\title{
Controversies on the Use of Radioiodine in Thyroid Cancer: We Need More and Better Data
}

\author{
Daniel A. Pryma \\ Division of Nuclear Medicine \& Clinical Molecular Imaging, Department of Radiology, Perelman School of Medicine at the \\ University of Pennsylvania, Philadelphia, Pennsylvania
}

See the associated articles on pages 1187 and 1195.

D ifferentiated thyroid cancer is a fascinating disease-it is both frustrating and heartening to treat. As eloquently pointed out by Tuttle in this issue of The Journal of Nuclear Medicine (1), "[M] ost patients with low-risk differentiated thyroid cancer will do exceptionally well, with disease-specific survival rates in excess of $99 \%$ and structural disease recurrence rates of less than $5 \%-10 \%$ after either low-intensity or high-intensity treatments." Conversely, patients do die of thyroid cancer, with an estimated U.S. mortality of 2,060 in 2018 (2). Although many thyroid cancer deaths are in patients with poorly differentiated or anaplastic cancers, we have all seen patients with mundane-appearing, seemingly low-risk disease ultimately succumb to thyroid cancer (3). Even the rosiest statistics show that patients with relatively favorable disease can experience morbidity and mortality. In this issue of The Journal of Nuclear Medicine, Schmidt et al. (4) clearly summarize the compelling, but incomplete, data suggesting that radioiodine therapy can positively alter the natural history of differentiated thyroid cancer in selected patients. Thus, regarding the application of radioiodine in thyroid cancer, there is a very clear potential for both overtreatment and undertreatment. Both can result in considerable morbidity and mortality and should, therefore, be avoided whenever possible. How do we decide the most appropriate treatment for any given patient? How do we determine whether a given patient is receiving the appropriate therapy or one too aggressive or not aggressive enough?

Regarding the potential for overtreatment of thyroid cancer with radioiodine, it is worthwhile to consider the following points.

First, even if the treatment is very well tolerated and has low toxicity, if there is no significant benefit, the risk-benefit ratio remains unfavorable. Said another way, if there is no benefit, even the most minor toxicity is unacceptable. Most patients with low-risk thyroid cancer are surgically cured and so the natural history of their disease cannot be improved by radioiodine therapy. The best

Received Jun. 18, 2018; revision accepted Jun. 19, 2018.

For correspondence or reprints contact: Daniel A. Pryma, Division of Nuclear Medicine \& Clinical Molecular Imaging, Department of Radiology, Perelman School of Medicine at the University of Pennsylvania, 3400 Spruce St., Donner 110-C, Philadelphia, PA 19104.

E-mail: dpryma@pennmedicine.upenn.edu

Published online Jun. 22, 2018.

COPYRIGHT (C) 2018 by the Society of Nuclear Medicine and Molecular Imaging. DOI: 10.2967/jnumed.118.214197 one can hope for is to alter a laboratory test value (thyroglobulin) to provide reassurance and facilitate management. This does certainly have value, but not the same value as prolonging (progression-free) survival.

Second, thyroid cancer is unlike other solid malignancies-features that confer high risk in many cancers do not necessarily do so in thyroid cancer. For example, a young patient even with extensive lateral neck nodal disease has stage I disease with a favorable prognosis. Furthermore, even patients with unfavorable features such as Hürthle-cell histology have a long median survival and relatively low disease-specific mortality. We cannot allow our experiences with other types of solid cancer to influence our impression of the risk of a patient with differentiated thyroid cancer.

Third, for most of the history of radioiodine use for thyroid cancer, there were no effective treatments for radioiodine-refractory disease. Therefore, it was reasonable to attempt radioiodine treatment even when it was highly likely to be futile. Today, there are several approved and investigational agents that have shown efficacy in these patients. Since most anticancer agents have some degree of overlapping toxicity (usually related to myelosuppression), it is important to avoid toxicity from futile treatments that may limit access to future potentially efficacious treatments. Therefore, in patients likely to have radioiodine-refractory disease, the aim should be to confirm this with the lowest possible therapeutic radioiodine dose.

Finally, in most cases, starting with a lower dose will not preclude additional treatment when needed. Although Schmidt et al. (4) point out that patients requiring a second treatment are likely to receive a higher cumulative dose than had they been administered a higher dose initially, such patients are only a small subset. Therefore, the total cumulative dose given to the population will be lower with an initial-low-dose approach. When long-term concerns are focused on stochastic events such as secondary malignancies, limiting the total population dose will be most effective in limiting the incidence of these statistically random events. Furthermore, there is evidence to suggest that no significant negative consequences will result from requiring a longer time to complete therapy (5); this consideration may also be important when patients require contrast-enhanced CT imaging for surgical planning.

Conversely, in contemplating the potential for undertreatment of thyroid cancer, several issues are worthy of deliberation.

First, when most patients do well, it is easy to fail to recognize the subset who will have poor outcomes and require more aggressive treatment. Although these patients can be difficult to identify in advance, they must not be ignored when detected. Appropriate care of thyroid cancer patients is nuanced and requires expertise. For example, Tuttle (1) rightly points out that 
data in support of lobectomy for larger tumors up to $4 \mathrm{~cm}$ requires proper patient selection to weed out the rare, less favorable cancers that will drive long-term morbidity and mortality. Ideally, in the future, additional clinical and genetic data will be used to help inform therapeutic decision making, as has been shown to be useful in breast cancer in the TAILORx trial (6). There is considerable work in this area regarding nodule characterization (7), but there are few data to date using this information to influence radioiodine treatment decisions.

Second, the data in support of low-intensity treatment, although quite strong, are relatively immature in light of the prolonged natural history of thyroid cancer. However, it is important to recognize that in the ablative setting, an undetectable stimulated thyroglobulin level is not a surrogate measure of success but the primary measure. After all, the goal of ablative therapy is the destruction of normal remnant thyroid tissue.

Third, adjuvant therapy has been shown to improve survival in several types of solid cancer, and there is no reason to think this should not hold true for patients with thyroid cancer and an elevated risk of disease-specific death. Identifying these patients with sufficient accuracy, though, remains challenging. Moreover, the utility of any particular activity (e.g., 1.1 GBq) in the ablative setting does not mean it will be appropriate in an adjuvant setting: thyroid cancer cells are likely to show less radioiodine uptake than normal thyroid tissue and will therefore require a higher activity to achieve a similar absorbed dose. In addition, when microscopic disease is present, a further increase in activity may be required to offset the reduced efficiency of energy absorption (when tumor deposits are smaller than the average range of $\beta$-particle emissions $[\sim 0.4 \mathrm{~mm}]$, the fraction of energy that is deposited within these deposits is reduced).

Finally, patients with iodine-avid structural metastatic disease are likely to benefit most from maximal tolerated doses (complete remissions from single submaximal treatments are rare). Therefore, a dosimetric approach to dose selection is likely optimal in this population. These are the patients most likely to have a clear and immediate benefit from radioiodine therapy and need safe but sufficient doses.

When contemplating the thoughtful and thought-provoking articles by Schmidt et al. (4) and Tuttle (1), it is vitally important to first consider that for a significant subset of patients, both articles advocate essentially equivalent approaches, with differences that may appear glaring to the experienced practitioner but that, in the grand scheme of a patient's life and medical journey, are quite minor. It attests to both the passion and the geekiness of thyroidologists and nuclear medicine physicians that we can argue vehemently over the difference between a single treatment of 1.1 or $2 \mathrm{GBq}$. The variability among such factors as patients' renal function, thyroid remnant volume, and hypothyroid versus recombinant human thyrotropin stimulation can easily overshadow the effect of a difference in administered dose on whole-body and individual organ radiation doses. Focusing on nuanced areas of disagreement distracts from the major issues that hamper our ability to best care for our patients.

Arguments and opinions can gain strength when not hampered by data. Although there are provocative studies representing wonderful work by thoughtful and dedicated researchers, there is no way around the fact that fundamental gaps in scientific knowledge limit our ability to understand optimal approaches to the use of radioiodine in thyroid cancer. Although it is absolutely true that clinical trial methodology has changed dramatically since
Dr. Hertz administered the first radioiodine therapy, it would be fatalistic to simply accept the inevitability of a persistent lack of data. Schmidt et al. (4) point out that, "To set up prospective randomized trials with radioiodine is not an easy task, requiring long-term follow-up because of the slowly growing tumor biology and the low event rate." Although it is true that the sample size and follow-up period to accurately detect the relative risk reduction of radioiodine therapy are quite large, they are feasible. For example, the National Oncologic PET Registry enrolled over 28,000 subjects in a single year (of an estimated 400,000 PET scans performed in the United States that year) $(8,9)$. In another example, the National Lung Screening Trial enrolled over 53,000 subjects and followed them for a median of $6.5 \mathrm{y}$ $(10,11)$. Overall, about $1 \%$ of the subjects died during followup, and the study was able to detect a $20 \%$ relative risk reduction in the experimental arm. I have illustrated imaging trials because they were performed largely in nuclear medicine or radiology departments in collaboration with referring clinicians and were successful. Furthermore, the forthcoming results of the ESTIMABL2 (12) and IoN (13) trials will greatly assist with accurate sample size calculations and expected accrual rates. Finally, among a large number of countries, the treatment of differentiated thyroid cancer is sufficiently uniform to make feasible a large, randomized international cooperative trial with a survival endpoint.

Tuttle (1) concludes his article by saying, "It is only through proactive, purposeful, and inclusive interdisciplinary cooperation that this field can be moved forward as we strive to optimize the intensity of therapy and follow-up for each individual with thyroid cancer." The latent linguist in me protests against the passive voice. This field will not be moved forward without our determined, active, and relentless demand to generate the data needed to enlighten these fundamental unanswered questions. Although many of us will no longer be practicing when these data mature in 10-20 y or longer, we should all strive to do our part to help develop the trials and prospective databases to gather such data for the benefit of future generations. To do so, we need to be open, honest, transparent, and scientifically rigorous. We cannot simply decry the events that led to our current situation. A well-known proverb states that the best time to plant a tree was 20 y ago; the next best time is now. Together, we should plant the trees that will someday grow into an improved understanding of the roles of radioiodine therapy for differentiated thyroid cancer.

\section{DISCLOSURE}

No potential conflict of interest relevant to this article was reported.

\section{REFERENCES}

1. Tuttle RM. Controversial issues in thyroid cancer management. J Nucl Med. 2018;59:1187-1194.

2. Siegel RL, Miller KD, Jemal A. Cancer statistics, 2018. CA Cancer J Clin. 2018;68:7-30.

3. Leite AKN, Cavalheiro BG, Kulcsar MA, et al. Deaths related to differentiated thyroid cancer: a rare but real event. Arch Endocrinol Metab. 2017;61:222-227.

4. Schmidt M, Görges R, Drzezga A, Dietlein M. A matter of controversy: is radioiodine therapy favorable in differentiated thyroid carcinoma? J Nucl Med. 2018;59:1195-1201.

5. Scheffel RS, Zanella AB, Dora JM, Maia AL. Timing of radioactive iodine administration does not influence outcomes in patients with differentiated thyroid carcinoma. Thyroid. 2016;26:1623-1629. 
6. Sparano JA, Gray RJ, Makower DF, et al. Adjuvant chemotherapy guided by a 21-gene expression assay in breast cancer. $N$ Engl J Med. June 3, 2018 [Epub ahead of print].

7. Nikiforova MN, Mercurio S, Wald AI, et al. Analytical performance of the ThyroSeq v3 genomic classifier for cancer diagnosis in thyroid nodules. Cancer. 2018;124:1682-1690.

8. Hillner BE, Siegel BA, Liu D, et al. Impact of positron emission tomography/ computed tomography and positron emission tomography (PET) alone on expected management of patients with cancer: initial results from the National Oncologic PET Registry. J Clin Oncol. 2008;26:2155-2161.

9. Hillner BE, Siegel BA, Shields AF, et al. Relationship between cancer type and impact of PET and PET/CT on intended management: findings of the national oncologic PET registry. J Nucl Med. 2008;49:1928-1935.
10. Aberle DR, Adams AM, Berg CD, et al.; National Lung Screening Trial Research Team. Reduced lung-cancer mortality with low-dose computed tomographic screening. N Engl J Med. 2011;365:395-409.

11. Aberle DR, Berg CD, Black WC, et al.; National Lung Screening Trial Research Team. The National Lung Screening Trial: overview and study design. Radiology. 2011;258:243-253.

12. Differentiated Thyroid Cancer: is there a need for radioiodine ablation in low risk patients? (ESTIMABL2). ClinicalTrials.gov website. https://clinicaltrials.gov/ct2/show/ NCT01837745. Published April 23, 2013. Updated May 16, 2017. Accessed June 20, 2018.

13. IoN: is ablative radio-iodine necessary for low risk differentiated thyroid cancer patients (IoN). ClinicalTrials.gov website. https://clinicaltrials.gov/ct2/show/NCT01398085. Published July 20, 2011. Updated October 26, 2016. Accessed June 20, 2018. 